\title{
Pembrolizumab, Pomalidomid und niedrig dosiertes Dexamethason bei rrMM
}

\begin{abstract}
Das Gesamtüberleben hat sich für Patienten mit multiplem Myelom in den letzten Jahren zwar verbessert, aber gegenüber Immunmodulatoren (IMiD) wie Pomalidomid refraktäre Patienten haben eine ungünstige Prognose. Können PD-1-Inhibitoren die durch Pomalidomid stimulierte Immunabwehr verstärken?
\end{abstract}

$\mathrm{n}$ einer offenen Phase-II-Studie erhielten 48 Patienten (median 3 Vortherapien) mit rezidiviertem oder refraktärem multiplem Myelom (rrMM) den PD1-Antikörper Pembrolizumab zusätzlich zu Pomalidomid und niedrig dosiertem Dexamethason. Die Mehrzahl der Patienten hatte bereits eine Therapie mit einem IMiD und einem Proteasominhibitor; 35 (73\%) waren gegenüber beiden Wirkgruppen refraktär, 31 (70\%) hatten eine autologe Stammzelltransplantation hinter sich, 30 Patienten (62\%) gehörten zu zytogenetischen Hochrisikogruppen.

Ein objektives Ansprechen zeigte sich bei 29 der 48 Patienten (60\%). 4 Patienten $(8 \%)$ erreichten eine komplette $\mathrm{Re}$ - mission (CR), 9 eine sehr gute partielle (VGPR, 19\%) und 16 eine partielle Remission (PR, 33\%). Die mediane Ansprechdauer betrug 14,7 Monate. Nach median 15,6 Monaten belief sich das mediane progressionsfreie Überleben (PFS) auf 17,4 Monate. Der Medianwert des Gesamtüberlebens wurde noch nicht erreicht. Vor Therapiebeginn entnommene Knochenmarksproben zeigten einen Trend für eine höhere PD-L1-Expression bei den Patienten mit Ansprechen und ein längeres PFS bei verstärkter Infiltration mit T-Lymphozyten.

Unerwünschte Ereignisse vom Grad 3/4 traten bei 19 der 48 Patienten auf, darunter bei $40 \%$ hämatologische Toxizitä- ten, bei $25 \%$ Hyperglykämien und bei $15 \%$ Pneumonien. Ein Patient verstarb infolge von Neutropenie und Sepsis. Als autoimmune Nebenwirkungen entwickelten 6 der 48 Patienten Pneumonitiden, 5 eine Schilddrüsenunterfunktion und 2 eine Nebenniereninsuffizienz. Die Patienten mit Pneumonitiden sprachen alle auf das Absetzen der Therapie an. Dosisreduktionen mussten insgesamt bei 22 Patienten durchgeführt werden (2-mal Pembrolizumab, 13-mal Pomalidomid, 7-mal Dexamethason). Bei Studienende hatten 22 Patienten einen Progress, 9 Patienten waren verstorben und 23 wurden weiter behandelt.

Fazit: Die Kombination von Pembrolizumab, Pomalidomid und niedrigdosiertem Dexamethason zeigte bei Patienten mit rrMM eine akzeptable Verträglichkeit und führte zu einem längeren Ansprechen.

Brigitte Schalhorn

Badros A et al. Pembrolizumab, pomalidomide and low dose dexamethasone for relapsed/refractory multiple myeloma. Blood. 2017; 130(10):1189-97.

\section{Myelom: Plus für autologe Transplantation}

\begin{abstract}
Für Patienten mit einem multiplen Myelom, das refraktär gegen Proteasomhemmer und Immunmodulatoren ist, gibt es bisher nur wenige Daten zum Nutzen der autologen Stammzelltransplantation. US-Onkologen haben nun überprüft, wie gut die Betroffenen tatsächlich davon profitieren.
\end{abstract}

D ie Onkologen vom MD Anderson Cancer Center in Houston werteten retrospektiv von 2000-2015 erhobene Befunde von 233 Patienten aus, die eine autologe Stammzelltransplantation (ASCT) erhalten hatten, nachdem sie weniger als eine partielle Remission auf die initiale Therapie gezeigt hatten. Die Teilnehmer wurden stratifiziert nach Einfach- bzw. Doppelrefraktärität. Das mediane Follow-up lag für die überlebenden Patienten bei 42 Monaten.

Insgesamt 105 der 233 Patienten sprachen weder auf Proteasomhemmer noch auf Immunmodulatoren an. Sie waren signifikant älter als die einfach refraktären Patienten ( 60 vs. 56 Jahre), und bei ihnen war häufiger eine Chemomobilisation von Stammzellen geglückt (51 vs.
$31 \%)$. Mehr Patienten in dieser Gruppe hatten eine Dreifachtherapie zur Induktion erhalten ( 52 vs. $21 \%$ ). Median hatten sie 2 Therapiezyklen vor der Transplantation bekommen, Patienten der anderen Gruppe dagegen nur einen Zyklus. Bei den Parametern „Gesamtüberleben“ und „progressionsfreies Überleben“ gab es zwischen den beiden Gruppen keinen signifikanten Unterschied.

Wie die Onkologen berichten, bewirkte die ASCT bei 188 Patienten (81\%) nach den Kriterien der International Myeloma Working Group ein mindestens partielles Ansprechen. Ein fast komplettes Ansprechen oder mehr stellten sie bei 52 Patienten (22\%) fest. Im Median lebten die Patienten noch 17,6 Monate ohne Progression; der Anteil der progressionsfreien Patienten lag nach zwei Jahren bei $38 \%$. Im Median überlebten die Patienten nach ASCT 48 Monate; nach 2 Jahren waren noch $74 \%$ am Leben, in der Gruppe der doppelt refraktären Patienten $71 \%$, in der anderen Gruppe $76 \%$. Ermutigend sei, dass die Patienten mit doppelt refraktärem Myelom nach Transplantation noch 14,4 Monate lang progressionsfrei gelebt hätten, so Lauren W. Veltri und Kollegen; in einer früheren Studie seien es nur 6,7 Monate gewesen. Sie halten es für möglich, dass dieser Effekt durch die Anwendung moderner Immunmodulatoren und Proteasomhemmer noch verstärkt wird.

Fazit: Patienten mit einem Myelom, das therapierefraktär gegen Proteasomhemmer und Immunmodulatoren ist, profitieren von einer ASCT. In der Studie lebten nach 2 Jahren noch 3 von 4 so behandelten Patienten.

Peter Leiner

Veltri LW et al. Outcome of Autologous Hematopoietic Stem Cell Transplantation in Refractory Multiple Myeloma. Cancer. 2017;123(18):3568-75. 